\title{
Dystonia in the joint hypermobility syndrome (a.k.a. Ehlers- Danlos syndrome, hypermobility type)
}

\author{
Hamonet $\mathrm{C}^{1,2 *}$, Ducret L ${ }^{3}$, Marié-Tanay $\mathrm{C}^{4}$, Brock $\mathrm{I}^{2}$ \\ ${ }^{1}$ Professeur éméritus Professor, Médical School of Créteil, University Paris-East-Creteil, 8 rue du Général Sarail, 91010 Créteil, France \\ ${ }^{2}$ Ehlers-Danlos Consultation, Hôtel-Dieu de Paris, 1 place du Parvis Notre-Dame, 75181, cedex 04 Paris \\ ${ }^{3}$ Department of Dermatology, Hôpital Tarnier, 89 rue d'Assas, 75006, Paris \\ ${ }^{4}$ Health Center of Argouges, rue de Beauvais, 14400 Bayeux
}

Received: January 01, 2016; Accepted: February 04, 2016; Published: Feburary 29, 2016

*Corresponding author: Hamonet C, Professeur éméritus Professor, Médical School of Créteil, University Paris-East-Creteil, 8 rue du Général Sarail, 91010 Créteil, France; Ehlers-Danlos Consultation, Hôtel-Dieu de Paris, 1 place du Parvis Notre-Dame, 75181, cedex 04 Paris ; E mail: pr.hamonet@wanadoo.fr

\begin{abstract}
Ehlers-Danlos syndrome first described by Tschernogobow (1896) in Moscow and Ehlers (1900) in Copenhagen is a mostly autosomal inherited genetic disease of collagen synthesis that sensitizes the ensemble of the connective tissue which becomes less resistant and less elastic. These two characteristics explain the symptomatology: fragility of the skin, of the vessels (haemorrhages) and the presence of a diffuse proprioceptive syndrome due to dysfunction of the receptors which are implanted into little or nonreactive connective tissue. Diagnosis of the hypermobile type of EDS is solely clinical as there is to date no genetic maker for the most frequent form of EDS. The rarity of the disease needs to be put into question before the crowd of patients at consultations. Our experience is based on an active database of 2212 patients which all fall under the Villefranche criteria. A great number of signs and symptoms have yet to be attributed to this syndrome. They are, combined with the unawareness of physicians about the syndrome, at the origin of therapeutic errors accompanied by the iatrogenic effects of prejudice towards these patients. This is the case of dystonia which is present in $75 \%$ of our cases. Dystonia plays an important part in the functional discomfort which is at the origin of a number of handicap situations. It seems to be related to dysautonomia common amongst the patients, proprioceptive problems and the multiple pains caused by the syndrome. Dystonia treatment with Amantadine and L-Dopa permits to obtain results which go further than the normally associated extrapyramidal treatment and opens new perspectives on the management of a syndrome that has been particularly difficult to treat.
\end{abstract}

Keywords: Ehlers-Danlos syndrome (EDS); Dystonia; Dysautonomia; L-dopa; Pains; Fatigue

\section{Introduction}

Ehlers-Danlos syndrome (EDS) recognition went through many vicissitudes since the first outstanding description by Tschernogobow [1] and Ehlers [2] respectively in Moscow in 1896 and in Copenhagen in 1900. EDS' genetic grounds have been recognized since 1949 [4] collagens role as early as 1956 [5]. EDS was studied in parallel by the rheumatologists (Brighton and Grahame) and the geneticist (Beighton) who is working mainly on articular hypermobility with different assessment tests. There is perfect similarity between the rheumatologists' joint hypermobility syndrome and the geneticists' EDS hypermobility type. These two denominations refer in fact to the same illness. However, a great body of clinical manifestations has not yet been assigned to this syndrome. They are, in combination with the physicians' usual unawareness of this syndrome, the cause of many diagnostic wavering with their iatrogenic side-effects that harm the patients. This is the case with dystonia.

\section{Material and Methods}

2,212 patients were diagnosed and followed up in the EhlersDanlos consultation in Paris, between 2006 and 2015. They were all examined by the same physician with the same evaluation grid both qualitative and quantitative allowing to rate from 0 to 4 the symptoms' subjective severity and objective data from clinical examination. The population's age varies from 2 years to 69 years (mean age: 32 ). $80 \%$ are women.

\section{Inclusion criteria}

All the patients in this study met the criteria of the geneticians' Villefranche classification [6].

On top of the criteria within this classification, we observed a group of 153 patients examined in 2013 with a quotation of severity equal or superior to $2 / 4$ (medium intensity) with clinical manifestations of the following: multiple pains (95\%), fatigue (93\%), proprioceptive problems (92\%), hemorrhages (93\%), GERD (72\%), bucco-dental manifestations (72\%), hyperacousia (75\%), diplopia (74\%), SOB (76\%), dysautonomia: heavy sweating (70\%), cold intolerance (74\%), a pseudo Raynaud with cold extremities (84\%), cognitive problems : attention (79\%) and memory (72\%). As of date there is no genetic test available for the hypermobile form of EDS. Finding other cases amongst the patient's family (95\%) is a strong diagnostic argument. 


\section{Dystonia identification}

Dystonia was diagnosed if a patient suffered from one or several of the following symptoms:

- Involuntary muscular contractions without movement such as fasciculation on the face, blepharospasm mainly, on the thigh, reminding of a mobile phone vibration in a trouser pocket,

- Sudden movements such as a fit of the wrist, the shoulders, the legs or wide movements which results in hitting objects or people or throwing off balance the patient for whom they occur in the lower limbs

- Trembling, jerking, hesitant hand movements

- Trembling fingers or thumbs in motion or at rest

- Muscular contractions often described as hardening of muscles, rigidity, constraining movement, or as cramps

- Lasting contractions in forced flexion of the thumb or fingers, in flexion and adduction of the feet,

- Writer's cramp when writing after variable amounts of time,

- Incessant, repetitive movement in flexion or extension of the foot and knee when sitting with feet on the ground,

- Repetitive movement of the trunk alternating between flexion and extension at the hip

- Diffuse tonic crises at the lower limbs with alternating violent movements worsened by tenting to immobilize them

\section{- Short contractions of the lower limbs leading to a fall}

- Partial or generalized tonic-clonic movements and the possibility of hematomas facilitated by the fine skin and the fragility of the vessels. These can be confused with seizure activity but the EEG remains normal

- Restless leg syndrome at night, which sometimes evolves into very violent jerks

- Bruxism, which we often encounter in EDS patients could be related to dystonia

These dystonic contractions provoke luxations of the shoulder, fingers, a hip, knee or the maxilla. They are most commonly of short duration but can prolong over several days, weeks, months or exceptionally years as we have observed in a few cases.

Dystonia is associated with the accentuation of other manifestations of the syndrome. Pain often increases to a very intense level in the part of the body where the dystonia occurs. Dysautonomic problems (vasomotor, sweating, tachycardia, orthostatic hypotension, freezing and cold intolerance, nausea, sensations of generally feeling unwell, POTS) at which Jaime Bravo [7] attaches fatigue. Pain itself can also provoke dystonia sometimes due to subcutaneous or intramuscular injections, traumatism, or simply during physical exam manoeuvre. It is perceived as painful by these hyperalgesic patients.

Dystonia exists in $75 \%$ of our patients with the following severity index: $2 / 4$ (39\%), 3/4 (29\%) and 4/4 (7\%).

\section{The treatment of dystonia within EDS}

Our therapeutic approach of EDS [8] centres on the amelioration of the proprioceptive troubles, of the pain as well as the fatigue. Foremost we use proprioceptive shoe inlays and particularly proprioceptive clothing specifically adapted for EDS, derived from the treatment of burn victims and oxygen therapy against fatigue, shortness of breath and migraines. For the last five years we have successfully used Amantadine after the discussion with Pierre Cesaro (neurologist, specialist in the treatment of Parkinson) [9].

When it was taken of the market in France we sought to replace it with L-Dopa which we prescribe at a low dosage (62, 5 mg q3d -Modopar: 50mg L-Dopa $+12,5 \mathrm{mg}$ Benserazide hydrochloride) adjusted to the needs of the patient especially in severe cases.

We describe here the case of a 54 year old woman, a family medicine physician, who had been diagnosed with EDS-HT. Signs present since childhood worsened at the age of 52: muscular pain, intense fatigue, proprioceptive problems manifesting most importantly with difficulties waling. Signs of dystonia could be observed in the lower limbs. She is very tired with crises of somnolence that disturb her professional life. The fatigue and muscular pain is partially alleviated by wearing proprioceptive shoe inlays, 3 sessions of oxygen therapy ( $3 \mathrm{~L} / \mathrm{min}) 20 \mathrm{~min} /$ day, baclofen and L-Carnitine.

The effects alleviate progressively over the course of 2 months a generalised sensation of muscular rigidity of the legs and face with difficulties in the articulations, muscular twitches increasing in frequency. She started progressively on a treatment with Modopar (62,5mg q3d). The results were spectacular; after 2 weeks she recuperated fluid motion in her legs, the involuntary muscular contractions disappeared, but there was also improvement in her vigilance and in the fatigue. She stopped the Baclofen without forfeiting anything in her muscular state. After 4 months of the treatment with L-Dopa the effects on her vigilance are maintained with the total loss of the hypersomnia. She can cut down her intake of Tramadol extended release threefold. Whereas before she thought about stopping all of her professional activities, she is now able to pursuit her professional life with efficacy. The oxygen therapy is maintained as well as the L-Carnitine for their action on muscles, because cutting down this part of the treatment lead to an increase in muscular pain.

\section{Discussion}

\section{Identifying dystonia as a frequent and evocative manifestation of EDS}

Dystonia should be looked for in any patient diagnosed with EDS. It even contributes to its diagnosis. On the other hand, when dystonia is present in a patient often associated with psychiatric 
problems one should think of the possibility of EDS as a diagnosis and inquire about the other evocative signs: diffuse overall pain, fatigue, hypermobility, cutaneous fragility, joint problems, hemorrhages and familial cases.

\section{Reflections on the pathophysiology}

We estimate that the alteration in proprioception plays a large part in the clinical manifestation of EDS. The receptors placed in a more elastic tissue, diminished in its thickness, easily deformed and compressed, having lost their elasticity (with a loss or attenuation of the elastic recoil),which do not or not well (not enough or too much) to solicitations. This is particularly true for the skin, which is the most important organ for the postural proprioception and for movement. This is also very true for the control of muscular activity via neuromuscular connections. This receptor dysfunction is also a plausible explanation for the anarchy within the autonomic nervous system, especially the crises of tachycardia and hypotension due to a loss in reactivity of the carotid sinus receptors implanted in altered collagen. This explanation can be expanded towards the mechanism causing dyspnea at effort, as the mechanoreceptors of the joints do not transmit the proper signals to the respiratory centers. One of the arguments in favor of this explanation is the positive effect special compressive clothing has on the proprioceptive control of the limbs (less falls, fewer luxation of the shoulder and fingers) and the improvement of the respiratory difficulties when wearing these clothes on the trunk. It is logical to interpret dystonia by way of the same mechanism and the poor information which is received by the specialized area of central nervous system. The positive effect observed by Roland Jaussaud [10] on a patient presenting with permanent, multiple, involuntary movements which completely ceased after starting to wear the special EDS compressive clothing. An association between dystonia and dysautonomia has often been made [11]. This corresponds to our observations made in consultation, especially in the instances that our patients came to call "their EDS crises". These crises are often accompanied by postural orthostatic hypotension (POTS) which plays an important role in the sensation of fatigue $[7,12]$. They are habitually painful, even very painful. These observations suggest an intricate pathophysiological relationship between dystonia, dysautonomia and pain in Ehlers-Danlos syndrome which become the main therapeutic focus.

\section{How to treat dystonia in EDS?}

The treatment of the proprioceptive problems (clothes and inlays), of the pain (inlays, local treatments to be preferred over the general route), of the fatigue (mostly oxygen therapy) and of the autonomic dysfunction by way of beta-blockers at low dose [13] seems to be a necessary prerequisite to be adjusted towards each patient individually. The specific treatment with anti-Parkinson medications have mostly been followed by their effects included on their effects in grand dystonic crises. The observation of positive effects outside of dystonia bears two questions: Firstly the role of dystonia itself on fatigue by way of less muscular activity and a better automatic control of movement, but also the role of dopamine as a molecule itself in this systemic disease.

\section{Conclusions}

Manifestations of dystonia in EDS is an important adjunct to further diagnosis and treatment, the understanding of its pathophysiology of this complex disease, which is little or poorly diagnosed, altering considerably life quality of the patients suffering from it and a source of many disabling situations.

The integration of all the manifestations of dystonia into the symptomatology of EDS enriches the clinic of this syndrome and furthers/advances new therapeutic perspectives in a particularly hard to treat pathology. L-Dopa seems to have positive effects that transcend those researched on dystonia itself.

\section{References}

1. Tschernogobow A, Cutis Laxa. Dermatologic and Venereology Society, Nov 13 (1891). Monatshefte für Praktische Dermatologie. 1892;14:76.

2. Ehlers E, Cutis laxa. Neigung zu Haemorrhagien in der Haut, Lockerung mehrerer Artikulationen (case for diagnosis), Dermatologishe Zeitschrift. 1901;8:173-174.

3. Johnson SAM, Falls HF. Ehlers-Danlos syndrome. A clinical and genetic study. Archives of Dermatology and Syphilology. 1949;60(1):82-105. doi:10.1001/archderm.1949.01530010085006.

4. Jansen LH. Le mode de transmission de la maladie d'Ehlers-Danlos. Journal de génétique humaine, 1954;204-218.

5. Tinkle BT, Bird HA, Grahame R, Lavallee M, Levy HP, Sillence D. The lack of clinical distinction between hypermobility type of EhlersDanlos syndrome and the joint hypermobility syndrome. Am J Med Denet A. 2009; 149A (11):2368-2370.

6. De Paepe A, Steinmann B, Tsipouras P, Wenstrup RJ. Ehlers-Danlos syndromes: revised nosology, Villefranche, 1997. Ehlers-Danlos National Foundation (USA) and Ehlers-Danlos Support Group (UK). Am J Med Genet. 1998;77(1):31-37.

7. Bravo JF. Elhers-Danlos syndrome (EDS), with special emphasis in the joint hypermobile syndrome. Revista medica de Chile. 2010;137(11):1488-1497. doi: /S0034-98872009001100013.

8. Hamonet C. Ehlers- Danlos Syndrome (EDS), a new clinical description, efficiency of physical medicine and rehabilitation. Six hundred individuals, of Physical and Rehabilitation Medicine. 2011;54 suppl1:e173.

9. Hamonet C., Césaro P. Syndrome d'Ehlers-Danlos et dystonie. Effet positif de l'Amantadine, La Presse médicale. 2014;43(9):1017-1018.

10.Jaussaud R. Premier colloque international francophone, les traitements du syndrome d'Ehlers-Danlos. Université Paris-EstCréteil. 2015.

11. Rowe PC, Barron DF, Calkins H, Maumenee IH, Tong PY, Geraghty MT. Orthostatic intolerance and chronic fatigue syndrome associated with Ehlers-Danlos syndrome. J Pediatr. 1999;135(4):494-499.

12. Bravo J. Dysautonomia in EDS-III, Premier colloque international francophone, les traitements du syndrome d'Ehlers-Danlos, Université Paris-Est-Créteil. 2015.

13. Amoretti R. Le cardiologue face au syndrome d'Ehlers-Danlos, Premier colloque international francophone, "les traitements du syndrome d'Ehlers-Danlos”, Université Paris-Est-Créteil. 2015. 